\title{
Ultra-High Energy Cosmic Rays and the GeV-TeV Diffuse Gamma-Ray Flux
}

\author{
Oleg E. Kalashev ${ }^{a}$, Dmitry V. Semikoz ${ }^{a, b}$, Günter Sigl $^{b}$ \\ a INR RAS, 60th October Anniversary pr. 7a, 117312 Moscow, Russia. \\ ${ }^{b}$ APC, 10, rue Alice Domon et Léonie Duquet, Paris 75205, France.
}

\begin{abstract}
Ultra-high energy cosmic ray protons accelerated in astrophysical objects produce secondary electromagnetic cascades during propagation in the cosmic microwave and infrared backgrounds. We show that such cascades can contribute between $\simeq 1 \%$ and $\simeq 50 \%$ of the GeV-TeV diffuse photon flux measured by the EGRET experiment. The GLAST satellite should have a good chance to discover this flux.
\end{abstract}

Introduction. Recently the HiRes collaboration established [1] the existence of the GZK cutoff [2]. If confirmed, this result suggests an astrophysical origin of ultra-high energy cosmic rays (UHECR).

There are two important contributions to secondary electromagnetic (EM) cascades from UHECR. One comes from the GZK process of pion production in interactions of UHECR protons with cosmic microwave (CMB) photons. Most of the energy transferred to photons, electrons and positrons in the subsequent pion decays would cascade down to $\mathrm{GeV}-\mathrm{TeV}$ energies, at which the Universe is transparent to photons. If the spectrum of primary protons is a power law $\propto E^{-\alpha}$, pion production energy losses and thus the energy deposited into EM cascades increases with decreasing power law index $\alpha$.

The second source of EM cascades is pair production by protons on low energy photons, $p+\gamma \rightarrow p+e^{+}+e^{-}$. This process is more efficient for steeper injection spectra with larger $\alpha$. Both processes together imply a minimal secondary photon flux at $\mathrm{GeV}-\mathrm{TeV}$ energies.

The goal of the present work is to study the contribution of EM cascades from UHECR proton interactions with background photons to the diffuse $\gamma$-ray background in astrophysical scenarios. We study the parameter space of UHECR models which fit the HiRes energy spectrum with the GZK cutoff. We show that the UHECR contribution to the $\gamma$-ray flux is in the range $1-50 \%$ of the diffuse flux measured by EGRET [4]. Relatively high values of this flux should enable the GLAST satellite [5] to disentangle it from other contributions such as from starforming galaxies [6], starbursts [7], large scale structure formation shocks [8], AGNs [9], blazars [10], and $\gamma$-ray bursts [11].

Modeling the primary proton flux. We parametrize the proton injection spectrum as $d N / d E \propto E^{-\alpha} \theta\left(E_{\max }-\right.$ $E)$, where $E_{\max }$ is the maximal proton energy and $\alpha$ is the power law index for which we consider the ranges $2 \times 10^{20} \mathrm{eV} \leq E_{\max } \leq 10^{21} \mathrm{eV}$ and $2 \leq \alpha \leq 2.7$, respectively.

We also consider the case of sources with variable density and/or luminosity. We assume the comoving source density to scale as $n(z)=n_{0}(1+z)^{m} \theta\left(z_{\max }-z\right) \theta\left(z-z_{\min }\right)$ where $m$ parameterizes the luminosity evolution. We consider the range $-2 \leq m \leq 4$, which practically in- cludes all astrophysical scenarios. The parameters $z_{\min }$ and $z_{\max }$ are the redshifts of the closest and most distant sources, respectively. We choose $z_{\min } \leq 0.01$ to avoid a GZK cutoff more pronounced than observed [1]. We fixed $z_{\max }=3$ which is large enough to take into account cosmologically distant sources. We note that both the luminosity evolution index $m$ and the maximum redshift $z_{\max }$ have similar qualitative influence on both proton spectrum and $\mathrm{GeV}-\mathrm{TeV}$ cascade fluxes.

For the rectilinear propagation of protons and cascades of secondary electrons, positrons and photons we used two independent codes 12, 13], which we compared on the level of individual interactions. For pion production by protons and neutrons both codes use the SOPHIA generator [14]. In addition, protons loose energy due to production of $e^{+} e^{-}$pairs, while neutrons decay. Secondary photons produce single and double pairs on the low energy photon background. Electrons and positrons interact via inverse Compton scattering and triplet pair production, and undergo synchrotron energy losses in extragalactic magnetic fields. Due to all those reactions secondary photons, electrons and positrons cascade down to $\mathrm{TeV}$ energies, where they are not affected by interactions with the CMB any more. However, even at these energies they can still interact with infrared (IR) and optical photon backgrounds. We use the recent model of Ref. [15] for these backgrounds.

Highly structured sources and large scale magnetic fields can lead to enhanced synchrotron fluxes up to $\mathrm{TeV}$ energies [16]. To be conservative we neglect magnetic fields and 3 dimensional effects here.

We fit the HiRes spectrum with the method described in Ref. 17]. Among all models characterized by $m, \alpha$, $E_{\max }, z_{\min }$, and $z_{\max }$ we choose those which fit the latest HiRes spectrum [1] at the $95 \%$ confidence level, taking into account empty bins above the highest energy events observed, as well as an energy uncertainty $\Delta E / E=17$ $\%$, which influences the shape of the spectrum around the GZK cutoff.

We consider the two main scenarios for the transition from a cosmic ray flux dominated by galactic sources to one dominated by extragalactic sources: In the "dip scenario" extragalactic protons dominate over the galactic contribution down to a few $10^{17} \mathrm{eV}$ and the dip observed 
in the energy spectrum between $\simeq 1$ and $\simeq 10 \mathrm{EeV}$ is caused by pair production by these protons [18]. We require predicted fluxes in this scenario fit the HiRes spectrum for $E \geq 2 \mathrm{EeV}$.

In the second scenario, the "ankle" in the spectrum around $\simeq 5 \times 10^{18} \mathrm{eV}$ is due to a cross-over from lowenergy galactic to high-energy extragalactic cosmic rays. Recent versions of this model include a mixed composition of UHECR at the highest energies [19]. To be conservative, in this case we fit the HiRes spectrum only for $E \geq 40 \mathrm{EeV}$.

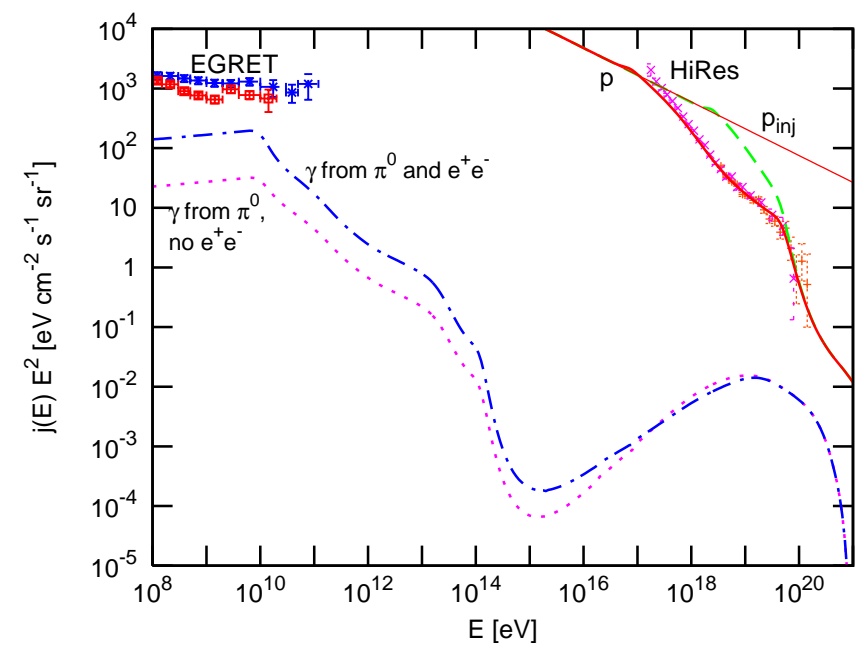

FIG. 1: Primary proton and secondary $\gamma$-ray fluxes for an injection spectrum $\propto E^{-2.45}$ up to $10^{21} \mathrm{eV}$ (shown as red, solid straight line marked $\left.p_{\text {inj }}\right)$ which evolves as $(1+z)^{3}$ between $z_{\min }=0$ and $z_{\max }=3$. In this case the UHECR flux is dominated by extragalactic protons down to a few $10^{17} \mathrm{eV}$ [18]. The lower red (solid) line is the proton flux, the blue (dash-dotted) line is the corresponding secondary $\gamma$-ray flux. The green (dashed) line is the proton flux without $e^{ \pm}$ production and the magenta (dotted) line is the corresponding $\gamma$-ray flux. The UHECR flux observed by HiRes [1] and two estimates of the extragalactic diffuse $\gamma$-ray background deduced from EGRET data as blue (higher) 3] and red (lower) crosses [4] are also shown.

The Diffuse GeV-TeV $\gamma$-ray flux. We now discuss the possible range of contributions of UHECR to the diffuse $\gamma$-ray flux in the EGRET band. In Fig. 1 we show a scenario where the dip is due to pair production by extragalactic protons. The lower red (solid) line was fitted to the HiRes spectrum [1] at energies $E \geq 2 \mathrm{EeV}$ and the corresponding EM cascade flux is shown as blue (dashdotted) line. Fig. 1 1 shows that practically all EM energy ends up in the $\mathrm{GeV}-\mathrm{TeV}$ region. By also showing the proton spectrum (green, dashed line) and the corresponding cascade flux (magenta, dotted line) when pair production by protons is neglected, Fig. 1 demonstrates that the $\mathrm{GeV}-\mathrm{TeV} \gamma$-ray flux is dominated by pair production losses of protons in this scenario.

The flux in energy carried by a differential spectrum
$j(E)$ is given by $\int E^{2} j(E) d \ln E$. One can then see from Fig. 1 that the UHECR energy lost to pair production by protons (energy flux difference between the red, solid and the green, dashed line) appears as the energy flux difference between the blue, dash-dotted and the magenta, dotted line, up to the pair flux which is not shown. Furthermore, the UHECR energy lost to pion production by protons (energy flux difference between the two red, solid lines) appears as the energy flux in the magenta, dotted line, up to the neutrino flux which is not shown.

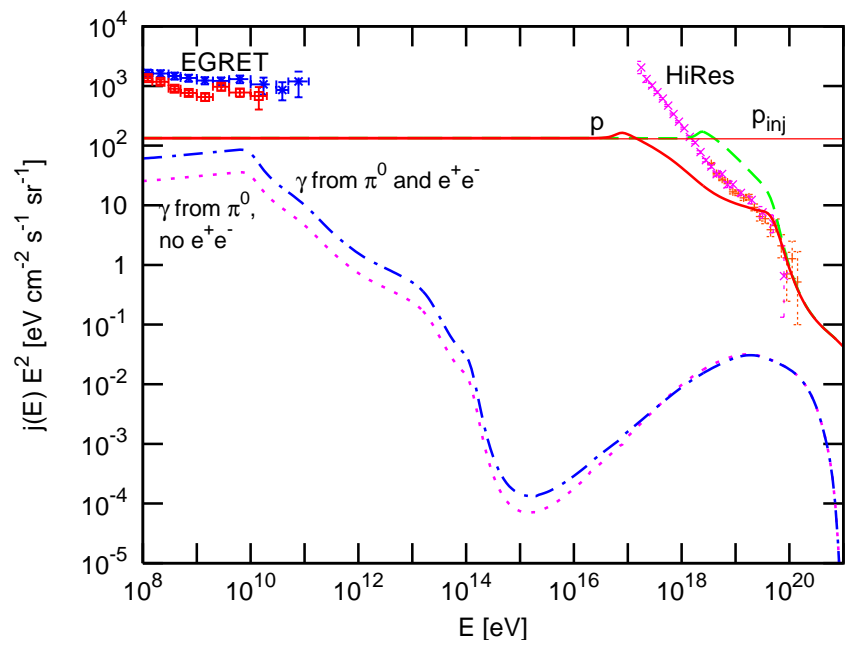

FIG. 2: Same as Fig. 1 but for a scenario with power law injection $\propto E^{-2}$ and evolution $\propto(1+z)^{3}$.

In Fig. 2 we show a scenario where the UHECR flux is extragalactic only above the ankle. In the particular case $\alpha=2$ one can see the relation between the energy deposited in $\mathrm{GeV}-\mathrm{TeV}$ photons and the energy lost by protons more directly. For this purpose we artificially continue the proton flux to low energies where it has the same order of magnitude as the secondary photon flux. Since protons loose similar amounts of energy to pion and to pair production in this scenario, the contribution of these processes to the $\mathrm{GeV}-\mathrm{TeV} \gamma$-ray flux is also comparable, contrary to the dip scenario of Fig. 1.

In Fig. 3 we show the range of possible contributions of UHECR interactions to the EGRET flux, as a function of redshift evolution index $m$. We express this as the fraction of integral fluxes between 1 and $2 \mathrm{GeV}$, where the EGRET energy flux $E^{2} j(E)$ is minimal. Fig. 3 shows that the diffuse $\gamma$-ray flux in the EGRET band strongly depends on the source luminosity evolution index $m$. In the ankle scenario, for a given value of $m$, it still depends on other parameters within a factor 3 , whereas in the dip scenario the scatter is much smaller due to partial degeneracy between $m$ and $\alpha$. All realistic astrophysical source distributions have $m \geq 0$, which implies that the contribution of secondary photons from UHECR will be at least $\simeq 1 \%$. For stronger evolution, $m=3-4$, this fraction increases to more than $50 \%$ of the EGRET flux. 


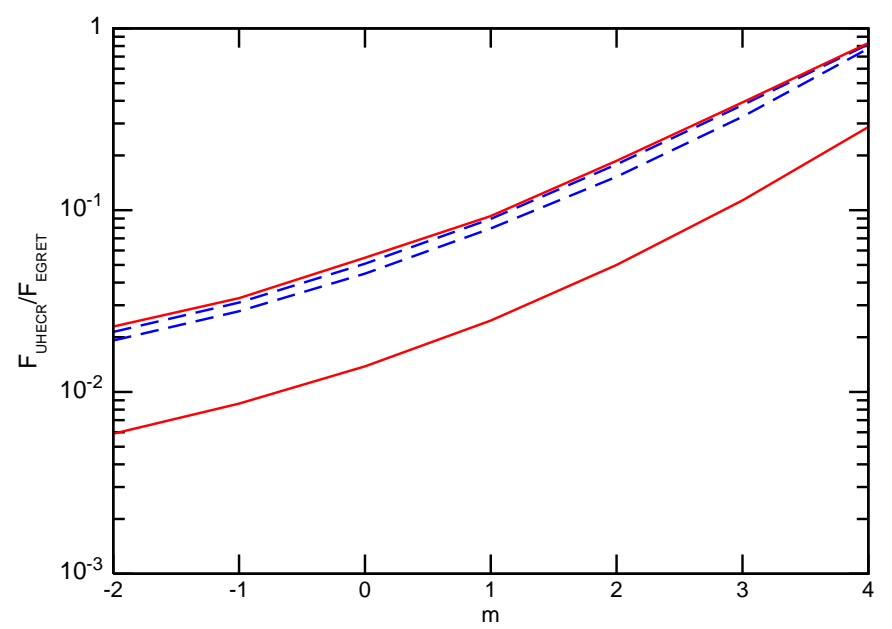

FIG. 3: Dependence on redshift evolution index $m$ of the minimal and maximal fractional contribution of UHECR interactions to the EGRET flux between 1 and $2 \mathrm{GeV}$. Blue, dashed lines are for fitting above $2 \mathrm{EeV}$ (dip scenario of the type shown in Fig. (1) and red, solid lines are for fitting the UHECR spectrum above $40 \mathrm{EeV}$.

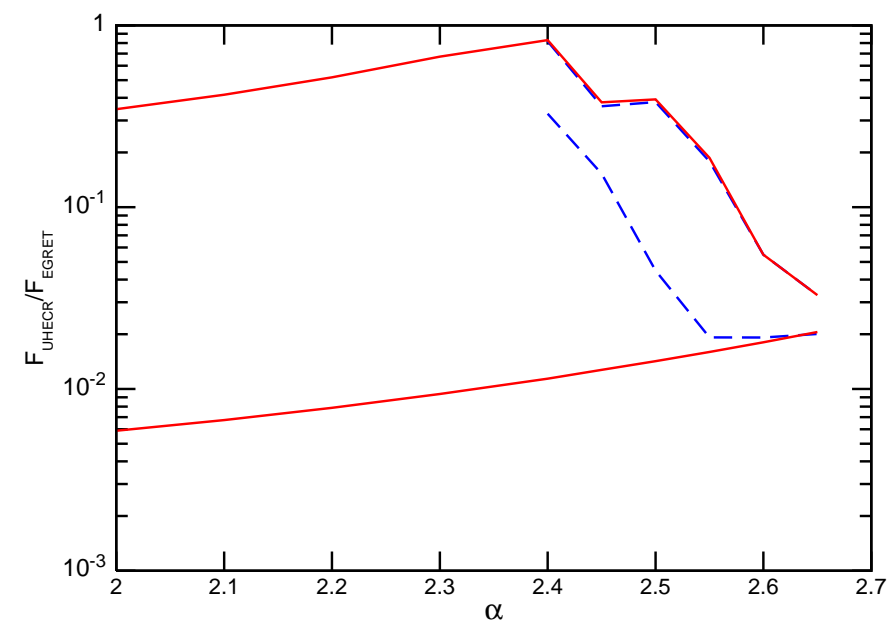

FIG. 4: As Fig. 3 but as function of the UHECR injection spectral index $\alpha$.

In Fig. 4 we show the range of possible contributions of UHECR interactions to the EGRET flux, as a function of the UHECR injection power law index $\alpha$. Contrary to the case of Fig. 3, the scatter is larger, especially for small values of $\alpha=2-2.4$. This is due to the strong dependence of the flux in the EGRET band on the value of $m$ for any given $\alpha$, see Fig. 3. The lower lines correspond to minimal values of $m=-2$, while the maximum is defined by $m=4$ for $\alpha \lesssim 2.4$, and by smaller values of $m$ for $\alpha \gtrsim 2.4$. Other parameter combinations would overproduce the cosmic ray flux below $\simeq 10 \mathrm{EeV}$.

In Fig. 5 we compare the range of EM cascade fluxes from UHECR with other possible astrophysical contributions in the EGRET band. Note that most of the

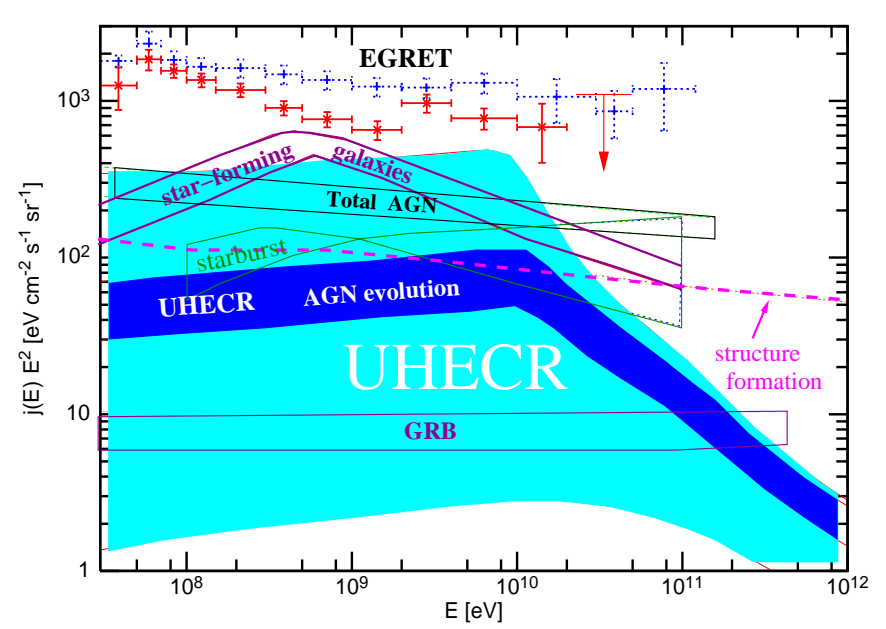

FIG. 5: The possible range of UHECR induced cascade fluxes (light shaded band) compared to estimated $\gamma$-ray fluxes directly produced by starforming galaxies [6], starbursts [7], large scale structure formation shocks [8], AGNs [9], and $\gamma$-ray bursts [1]. The dark shaded band shows the range of EM cascade fluxes from UHECR sources evolving as AGN [21]. The extragalactic diffuse $\gamma$-ray background from EGRET is as in Fig. 1]

uncertainty of the UHECR cascade flux comes from the unknown source evolution. The scatter for a given evolution such as for AGN is thus much smaller, as seen from Fig. 5. We remark that dark matter annihilations may also contribute to the diffuse flux 22].

The GeV-TeV cascade flux in scenarios where extragalactic cosmic rays dominate down to below the ankle at $\simeq 5 \times 10^{18} \mathrm{eV}$ would be practically diffuse for an instrument such as GLAST which will be sensitive to anisotropies down to $\sim 0.1 \%$ [20]. This is because the cosmic ray flux below the ankle is dominated by cosmological sources and is isotropic at the percent level, and because large scale magnetic fields should lead to significant additional isotropization of primary protons [23] and secondary pairs.

In contrast, lacking statistics, UHECR anisotropies at the $10 \%$ level can currently not be ruled out at energies around or above the GZK cutoff, $E>40 \mathrm{EeV}$, because sources of such UHECR may have a small density $\sim 10^{-5} \mathrm{Mpc}^{-3}$ [24]. Furthermore, at energies $E \sim$ $200 \mathrm{GeV}-10 \mathrm{TeV}$ the $\gamma$-ray absorption length in the IR background becomes small compared to the Hubble radius, but still large compared to the $\sim 10 \mathrm{Mpc}$ length scales of pion production and EM cascade development around the source. This is also reflected in the amount of $\gamma$-ray flux suppression in Figs. 1 and 2. Independent of the poorly known size of deflection, this could lead to considerable correlation with nearby UHECR sources, as in the case where discrete sources emit very high energy $\gamma$-rays directly [25]. In scenarios such as in Fig. 2. where the secondary cascade flux is dominated by pion 
production due to relatively hard UHECR injection spectra, this flux could, therefore, exhibit detectable small scale anisotropy around $\sim 200 \mathrm{GeV}$.

Conclusions. UHECR interactions with low energy photons can significantly contribute to the observed diffuse flux of $\gamma$-rays at energies between $\sim 100 \mathrm{MeV}$ and $\sim \mathrm{TeV}$. In this paper we studied the dependence of this contribution on unknown parameters of astrophysical UHECR scenarios. We found that UHECR contribute no less than $1 \%$ to the observed EGRET flux, and up to $50 \%$ in some cases. This suggests that the GLAST satellite, which at $\mathrm{GeV}$ energies will be $\simeq 30$ times more sensitive to point sources than the EGRET experiment, will likely be sensitive to the UHECR induced contribution. Even ground-based instruments such as HESS and the future CTA may be sensitive to the cascade flux between $\simeq 0.1$ and $\simeq 10 \mathrm{TeV}$, although such experiments are less well suited for diffuse backgrounds.

If sources of extragalactic highest energy cosmic rays are rare and dominate the flux down to only $\simeq 5 \times$ $10^{18} \mathrm{eV}$, the cascade background may have significant anisotropy at energies around $200 \mathrm{GeV}$.

To summarize, future measurements of resolved and unresolved components of the diffuse EGRET $\gamma$-ray background or upper limits on such components can give important information on UHECR origin and the distribution of their sources.

Acknowledgments. We thank F. Stecker for providing us with tables for the IR/optical backgrounds for the model of Ref. [15]. The numerical simulations were performed at the computer cluster of the Theory Division of INR RAS. O.K. acknowledges financial support from in2p3/CNRS for a collaboration visit at APC, Paris.

[1] R. Abbasi et al. [HiRes Collaboration], arXiv:astro-ph/0703099.

[2] K. Greisen, Phys. Rev. Lett. 16, 748 (1966); G. T. Zatsepin and V. A. Kuzmin, JETP Lett. 4, 78 (1966) [Pisma Zh. Eksp. Teor. Fiz. 4, 114 (1966)].

[3] P. Sreekumar et al., Astrophys. J. 494, 523 (1998) astro-ph/9709257.
[4] A. W. Strong, I. V. Moskalenko and O. Reimer, Astrophys. J. 613, 956 (2004) arXiv:astro-ph/0405441.

[5] For general information see http://www-glast.stanford.edu

[6] V. Pavlidou and B. D. Fields, Astrophys. J. 575, L5 (2002) arXiv:astro-ph/0207253.

[7] T. A. Thompson, E. Quataert and E. Waxman, Astrophys. J. 654, 219 (2006) arXiv:astro-ph/0606665.

[8] U. Keshet, E. Waxman, A. Loeb, V. Springel and L. Hernquist, Astrophys. J. 585, 128 (2003) arXiv:astro-ph/0202318.

[9] C. D. Dermer, arXiv:astro-ph/0605402

[10] F. W. Stecker and M. H. Salamon, Astrophys. J. 464, 600 (1996) arXiv:astro-ph/9601120.

[11] C. D. Dermer, arXiv:astro-ph/0610195.

[12] E. Armengaud, G. Sigl, T. Beau and F. Miniati, arXiv:astro-ph/0603675 see http://apcauger.in2p3.fr//CRPropa.

[13] O. E. Kalashev, V. A. Kuzmin and D. V. Semikoz, arXiv:astro-ph/9911035. Mod. Phys. Lett. A 16, 2505 (2001).

[14] A. Mucke, R. Engel, J. P. Rachen, R. J. Protheroe and T. Stanev, Comput. Phys. Commun. 124, 290 (2000) arXiv:astro-ph/9903478.

[15] F. W. Stecker, M. A. Malkan and S. T. Scully, Astrophys. J. 648, 774 (2006) arXiv:astro-ph/0510449.

[16] E. Armengaud, G. Sigl and F. Miniati, Phys. Rev. D 73, 083008 (2006).

[17] G. Gelmini, O. Kalashev and D. V. Semikoz, arXiv:astro-ph/0702464

[18] V. Berezinsky, A. Z. Gazizov and S. I. Grigorieva, Phys. Rev. D 74, 043005 (2006) arXiv:hep-ph/0204357; astro-ph/0210095 Nucl. Phys. Proc. Suppl. 136, 147 (2004) astro-ph/0410650; Phys. Lett. B 612 (2005) 147 astro-ph/0502550.

[19] D. Allard, E. Parizot and A. V. Olinto, Astropart. Phys. 27, 61 (2007) arXiv:astro-ph/0512345.

[20] D. Hooper and P. D. Serpico, arXiv:astro-ph/0702328.

[21] E. Waxman and J. N. Bahcall, Phys. Rev. D 59, 023002 (1999) arXiv:hep-ph/9807282.

[22] D. Elsaesser and K. Mannheim, Phys. Rev. Lett. 94, 171302 (2005) arXiv:astro-ph/0405235.

[23] G. Sigl, F. Miniati and T. Ensslin, Nucl. Phys. Proc. Suppl. 136, 224 (2004) arXiv:astro-ph/0409098.

[24] M. Kachelriess and D. Semikoz, Astropart. Phys. 23, 486 (2005) arXiv:astro-ph/0405258.

[25] A. Cuoco, S. Hannestad, T. Haugbolle, G. Miele, P. D. Serpico and H. Tu, arXiv:astro-ph/0612559. 